\title{
La integración de enfoques de enseñanza como vía para elevar la motivación por la estimación de proyectos de software en estudiantes de Ingeniería Informática
}

\author{
Walfredo González Hernández. Universidad de Matanzas (Cuba) \\ Recepción: 24 de marzo de 2016 | Revisión: 28 de marzo de 2016 | Aceptado: 11 abril de 2016 \\ Correspondencia: walfredo.glez@umcc.cu \\ Citar: Gonzalez Hernandez, W. (2016). La integración de enfoques de enseñanza como vía para elevar la motivación por la \\ estimación de proyectos de software en estudiantes de Ingeniería Informática. ReiDoCrea, 5, 78-89.
}

\begin{abstract}
Resumen: La motivación por la estimación de los proyectos de software es parte de la formación profesional del ingeniero informático. En este sentido, debe definirse las dimensiones e indicadores de este concepto así como las vías fundamentales para su desarrollo. En este artículo se asumen como vías para lograrlo la integración de los enfoques de la enseñanza: sistémico, problémico y aprendizaje basado en proyectos. Posteriormente se presenta la validación de la alternativa.
\end{abstract}

Palabras Claves: Motivación | Aprendizaje basado en proyectos

The Integration of Focuses of Teaching Like Road to Raise the Motivation for the Estimate of Projects of Software in Students of Information-Technology Engineering

\begin{abstract}
The motivation for the estimate of the projects of software is a part of the technical training of the information-technology engineer. In this sense, dimensions and this concept's indicators as well as the fundamental roads for your development should be defined. The author assumes like manners to achieve the integration of the focuses of the teaching in this article: Systemic, probllematic and learning based in projects. At a later time the validation of the alternative shows up.
\end{abstract}

Keywords: Motivation | Project Based Learning

\section{Introducción}

La informática como ciencia ha recorrido un largo camino desde sus inicios hasta el momento que se expresa en la actividad que se realiza. El nivel de penetración de la informática en las diferentes ramas del saber humano ha llevado a grandes cambios en estas y en la ejecución de extensos proyectos de informatización.

Uno de los momentos más integradores de la formación de un profesional informático en el proceso de desarrollo de un sistema se focalizan en el proceso de estimación de un proyecto. En este proceso se desarrollan diferentes abstracciones del sistema a desarrollar en dependencia del modelo de estimación seleccionado. Si se estima utilizando puntos de función entonces se toma como base las funcionalidades del sistema escritas en uno de los tres modelos conocidos. Posteriormente se comienza a seguir un conjunto de acciones de transformación de estos procesos para obtener las proyecciones necesarias.

Existen diferentes modelos de estimación de proyectos de software. Entre los más destacados en la literatura se encuentran COCOMO II, SLIM y Puntos de Función. Cada uno de ellos presenta un proceso de estimación completamente diferente ajustado a sus requerimientos. También es importante destacar que cada uno de ellos puede ser utilizado en diferentes estadíos del desarrollo para estimar. Las estimaciones por puntos de función son interesantes porque permiten obtener los costos, el esfuerzo y el tiempo de desarrollo desde los primeros estadíos del desarrollo. Aun cuando estas estimaciones son groseras en cuanto a la exactitud de sus resultados, permiten un acercamiento del gestor de proyectos a la organización del proyecto. Posteriormente puede utilizar otros modelos para la estimación más 
certera o fina de estos valores. En este momento entran a jugar su papel modelos como COCOMO II.

Sin embargo, la importancia de los modelos de estimación se ve disminuida en los estudiantes por la complejidad de los cálculos que deben ser realizadas. Existen diversos sistemas para hacerlos pero todos estos son propietarios y no tienen licencias para universitarios. En la universidad se ha realizado un sistema para los cálculos de las estimaciones, sin embargo los estudiantes no reflejan motivación por los procesos de estimación.

De esta manera se concluye que la estimación es una actividad importante dentro del proyecto al marcar las pautas del desarrollo de un sistema, sin embargo los sistemas no es el único resultado de la actividad informática. Esta informatización de procesos organizacionales puede darse desde varias actividades desde las disciplinas científicas de la informática como ciencia y que pueden integrarse o no en la solución de la problemática.

\section{Desarrollo}

La medición es parte consustancial de los procesos de informatización en los cuales puede desempeñarse cualquier profesional de la informática. Son muchas las cuestiones que se pueden medir en estos procesos destacándose las medidas directas e indirectas. Una de las medidas indirectas más importantes destacadas en la literatura actual (Biafore, 2011; Dawson, 2009; González Hernández, 2013a; Oman, Tumer, Wood, \& Seepersad, 2013) son las estimaciones.

La estimación es un proceso orientado al futuro pues se trata de determinar el costo, el tiempo y el esfuerzo en el desarrollo de un sistema antes de su ejecución. Para (Pressman, 2010), la estimación es el proceso de medición anticipada de la duración, esfuerzos y costes necesarios para realizar todas las actividades y obtener todos los productos asociados a un proyecto. Es necesario tener en cuenta numerosos aspectos que afectan a la estimación como la complejidad del proyecto, su estructuración, el tamaño, los recursos involucrados y los riesgos asociados. Para ello es necesario poseer una vasta experiencia en el desarrollo de sistemas.

Como se puede inferir de la definición anterior, la estimación es un proceso del cual depende en gran medida la asignación de recursos humanos y materiales a un proyecto así como su planificación. La experiencia de la organización en esta tarea así como la documentación exhaustiva de sus procesos de estimación anteriores es decisiva en este proceso.

Una de las estimaciones precisamente se basa en la búsqueda de un experto que basado en su experiencia determine cuánto puede demorar y el coste del sistema que se pretende desarrollar. Sin embargo, esta estimación no es la más adecuada debido a las imprecisiones de la experiencia humana o por no contar con un experto para realizarla. Una de las opciones está en las estimaciones tempranas, como es el caso de estimaciones por puntos de función, que permiten obtener estos valores a partir del levantamiento de requisitos. Esta estimación adolece de una dificultad, al ser en estadíos tempranos del proyecto puede tener resultados no muy exactos. Otra de las estimaciones más utilizadas en el mundo es COCOMO II como modelo algorítmico en el cual se pueden realizar estos cálculos en tres niveles. Estos modelos pueden combinarse para ofrecer estimaciones más exactas en la medida que el proyecto avanza y se necesiten realizar ajustes. También son necesarios en procesos iterativos e incrementales para cada una de las iteraciones que se van a ejecutar en el proyecto. 
Cada uno de estos modelos de estimación debe ser utilizado en dependencia del momento en el cual se necesite la estimación o del proyecto que se va a desarrollar. Algunas de las metodologías, como XP (Extreme Programming) o RUP (Rational Unified Process) poseen ya modelos de estimación propios derivados de estos clásicos. Determinar con claridad y precisión cuál modelo utilizar y el momento más adecuado para cada proyecto en dependencia de las necesidades es un proceso que necesita de experiencia en el rol de gestor de proyectos.

Una de las problemáticas que tienen estos modelos de estimación es la gran cantidad de cálculos que deben realizar los estudiantes y la múltiple cantidad de criterios que deben ser tenidos en cuenta. Otro de los aspectos que hace ser poco interesante para ellos se encuentra en la pobre comprensión de la importancia de la estimación de proyectos a partir de la tendencia a la ejecución detectada en ellos (González Hernández, 2004, 2013; ; González Hernández \& Estrada Sentí, 2014). Estos conocimientos se imparten durante el quinto año de la carrera en la cual ellos deben finalizar sus estudios con un trabajo de investigación en el cual desarrollan un software. Para ello deben estimar el proyecto de software y resulta interesante que durante la asignatura ellos estimen el costo, el tiempo de duración y el esfuerzo que debe emplearse para el desarrollo.

\section{Motivación por la estimación de proyectos de software}

Una vez explicitado el contenido de enseñanza informático en el curso es necesario abordar la motivación en los estudiantes. En forma en que la personalidad elabora la necesidad encontrando su expresión diferentes manifestaciones concretas (reflexión, valoración, comportamiento) dando sentido, fuerza y dirección a la personalidad. Es interno, tiene diferentes formas de expresión, la personalidad conforma el motivo incidiendo sobre la necesidad (Autores, 2003). Para otros autores (González Rey, 2010; Lin, 2012), el motivo se aborda como todo aquello que estimula y conducen la actividad del hombre con vista a satisfacer sus necesidades, es decir, todo en lo que ha encontrado su "encarnación" la necesidad. Quiere decir que debe existir una necesidad por aprender a estimar para que se oriente esta actividad de aprendizaje de esta ciencia. Esta necesidad es posible de crear a partir de su posible incorporación a la universalización de la educación superior y, muy importante, como parte de su desempeño profesional como ingenieros. Ello conlleva a estos estudiantes a ver la estimación como parte del sistema de contenidos que deben dominar para desarrollar con éxito procesos de desarrollo de sistemas en la organización en las cuales se desempeñen. Por ende, comienzan a integrar estos contenidos nuevos a su proyección futura como ingenieros informáticos lo cual favorece la aparición de la motivación por ellos. En la medida en que se van integrando las clases sobre la estimación y sus proyectos van perfilando procesos de desarrollo van adquiriendo estos contenidos como parte de su preparación profesional.

Por tanto en esta proyección futura se expresa la motivación y aspiraciones futuras que manifiestan los estudiantes al estimar, como un sistema de clases en correspondencia con sus proyectos de vida como ingenieros industriales durante el proceso de enseñanza - aprendizaje de la estimación de proyectos. Por ello se podría asumir como indicadores de esta motivación profesional:

1. Expresan niveles de interés y aspiración futura en la construcción y ensayo de estimaciones de proyectos de sistemas para su formación personal y colectiva al elaborar estimaciones como parte de un proyecto futuro durante el proceso enseñanza-aprendizaje de estos modelos. 
2. Identifican la estimación como una de sus posibles esferas de actuación dentro de su imagen anticipada de las funciones de un ingeniero informático.

3. Expresan niveles de autonomía e iniciativa, al seleccionar el modelo de estimación e implantarlo como parte de un proyecto futuro.

4. Manifiestan niveles de satisfacción personal al elaborar las estimaciones como parte de un proyecto futuro.

5. Se apropian de manera activa y consciente de los contenidos de la estimación de proyectos aplicándolos a su sistema a desarrollar.

Ya clarificado el qué se va a enseñar en este proceso de preparación pedagógica, es imprescindible el análisis de cómo se va a enseñar este sistema de contenidos para lograr motivar al estudiante. La introducción de la enseñanza problémica ha sido una vía con demostrada efectividad en el contexto de la clase (Medina, 2012; Velázquez, Pérez, \& Rodríguez, 2012). Sin embargo, otros autores le reconocen que no es pertinente cuando se quiere motivar por un sistema de contenidos generalizador (González Hernández, 2013b; González Hernández, Estrada Sentí, \& Martínez Llantada, 2006). En estos autores se realiza una propuesta para la enseñanza de la informática que pudiera ser llevada a la enseñanza de la estimación de proyectos La propuesta, en esencia, aborda la integración de tres enfoques de enseñanza: el enfoque de sistema, el enfoque de proyectos y el enfoque problémico.

Cuando se aborda el enfoque de sistema, pensado para la enseñanza de la informática (González Hernández et al., 2006), se plantea el cómo preparar a los estudiantes desde una familia de sistemas a otra. Extrapolando la concepción sistémica al asunto de este artículo, se hace necesario destacar lo importante de estructurar los diferentes modelos de estimación de proyectos como eslabones del proceso. Estos eslabones van preparando el paso para el posterior desde las propias opiniones de los estudiantes sobre las clases recibidas. El enfoque sistémico es propio de concepciones generales asociadas a cursos. Este proceso propicia el desarrollo de la independencia cognoscitiva desde la perspectiva de la crítica y la asunción de los errores cometidos.

Otro de los enfoques importantes a tener en cuenta es el enfoque de proyectos. Este enfoque propuesto (Expósito Ricardo, 2009; González Hernández, 2013b; Segura Montero \& González Hernández, 2015) es una adecuación del aprendizaje basado en problemas para la enseñanza de la informática. Es entonces necesario realizar una breve panorámica del aprendizaje basado en problemas que revele la esencia de su teoría.

Para (Sabaté \& Valero-García, 2012, pág. 2) “... consiste en plantear a los alumnos un proyecto que sea percibido por ellos como ambicioso pero viable, que deben llevar a cabo en pequeños equipos. El proceso de enseñanza y aprendizaje se organiza entonces en función de las necesidades de aprendizaje de los equipos." Si bien se plantea por varios autores (González-Jorge, Roca, Torres, Armesto, \& Puente, 2014; López \& Vázquez, 2013) la utilización de equipos en contextos tecnológicos por la propia naturaleza del proceso, para la planificación de la clase puede realizarse de manera individual. Esta afirmación no contradice la idea del trabajo colaborativo en equipos sino que deja este para el proceso de construcción del contenido didáctico y no ser necesario en el proceso de planificación de la clase.

Para varios autores (Peña, Garrido, \& López, 2015; Rivera Julio \& Turizo Martínez, 2014) los problemas que se asumen en este contexto de aprendizaje son de naturaleza interdisciplinarios con lo cual no se concuerda asumiendo una postura multidisciplinaria. "La multidisciplinariedad se impone en todas aquellas partes donde 
las especialidades están institucionalizadas en sectores especializados, cuya coordinación es al mismo tiempo elaboración de una síntesis específica de las informaciones heterogéneas y especiales, siendo el objetivo, en todas partes y siempre, un objetivo de acción."(Hernández, 2009). La integración de profesionales de diversas especialidades en la solución de problemáticas complejas es la única vía posible para lograrlo (Maldonado, 2009, 2014).

La multidisciplinariedad, como parte del paradigma complejo en la comprensión de la realidad, es una necesidad para poder comprender al mundo en forma compleja, para poder resolver problemas los cuales las causas se transforman en efectos y viceversa, para poder pensar una realidad que desestructura especializaciones con un alto grado de desconexión entre ellas, las relaciones del hombre con el mundo y las interrelaciones del mundo, porque no se da el hombre aislado, como tampoco se da la realidad como cosas aisladas.

La estimación de proyectos, vista desde la perspectiva didáctica, como contenido a enseñar es un proceso multidisciplinario. La integración de diversas disciplinas informáticas como redes, programación y gestión de proyectos en el sistema de ítems de complejidad, de ambiente entre otros. En este proceso se analizan diferentes contenidos que no son estudiados en la carrera como otros lenguajes de programación, factores de sistemas distribuidos, entre otros, que no son parte de su contenido escolar. Sin embargo, si se quieren prepararlos para enfrentar este proceso se deben tomar en cuenta estos elementos y propiciar una formación multidisciplinaria. Esta concepción de la estimación, y del sistema de contenidos que ellos suponen, llevan a una integración de varias especialidades profesionales dentro de su perfil y las interrelaciones que este puede llevar con otras especialidades. Esta es la causa del por qué en este artículo no se considera la interdisciplinariedad como el eje fundamental sino parte integrante y condición necesaria para el logro de la multidisciplinariedad.

Una vez definido el carácter multidisciplinar de los problemas que se pueden presentar en este contexto, es necesario determinar las posiciones acerca de la determinación de estos problemas. Para algunos autores (Guitart, 2011; Hanafy Morsy et al., 2015; McGibbon \& Belle, 2015; Parra Castrillón, Castro Castro, \& Amariles Camacho, 2014) en el aprendizaje basado en problemas quien los determina es el profesor y para otros (González Hernández, 2013b; González Hernández 2013; Kanhime Kasavuve \& González Hernández, 2014; Rivera Julio \& Turizo Martínez, 2014; Rodríguez González \& Fernández Batanero, 2015) los determina el estudiante en interacción con el profesor, este último para determinar si se corresponden con el objetivo que se pretenda evaluar. Este artículo se asume una solución intermedia. Al ser el objetivo del curso la estimación de su proyecto de software para trabajo de investigación este objetivo es consensuado con el estudiante, mientras que el contenido de enseñanza en la clase es seleccionado por el profesor. El estudiante selecciona el contenido de la clase. De esta manera se garantiza la motivación del aprendizaje por el estudiante.

Una vez concluida esta etapa, entonces se pasa al tratamiento de los diferentes modelos de estimación. En este proceso se toma la clase de un estudiante como situación problémica para introducir el nuevo contenido necesario para la planificación de la estimación utilizando cada modelo. Cada una de las clases que imparte el profesor se interrelaciona con las demás a partir del lugar y el papel que desempeñan los diferentes modelos y sus partes integrantes en la gestión del proyecto. Cada uno de estos modelos surge para dar respuesta a diferentes proyectos o a momentos diferentes en cada proyecto que permite integrarlos al proyecto del estudiante en diferentes momentos aunque sean introducidos en una clase diferente tomada de los 
proyectos de los estudiantes, siempre teniendo en cuenta el objetivo de la propuesta. De esta manera se garantiza la transferencia de conocimientos y habilidades a situaciones docentes nuevas, lo que se traduce en aprendizaje como producción de sus propios y nuevos saberes, incluyendo en los mismos la actividad metacognitiva del estudiante en su aprendizaje.

Con este tratamiento a la estimación de proyectos se logra que el estudiante asuma el lugar protagonista y sujeto en el proceso, tornándose constructor y reconstructor de sus saberes, desarrolla un pensamiento analítico, reflexivo, crítico y alternativo que se materialice en un nuevo estilo de aprendizaje, de manera que el proceso cognitivo se transforme de reproductivo, concreto y situacional en uno productivo, generalizador y conceptual, construir para sí, además de conocimientos sobre el mundo externo y objetivo, conocimientos sobre su aprendizaje y su propia personalidad, necesidades, vías y formas de actuar (metaconocimientos), entre otras cuestiones.

Para el autor de este artículo es importante destacar que los problemas a presentar a los estudiantes en la enseñanza de la informática se coincide con (González Hernández, 2013b; González Hernández 2013; González Hernández et al., 2006; Kanhime Kasavuve \& González Hernández, 2014) cuando lo definen como: “... aquella situación contradictoria que no tiene una vía de solución determinada, incluyendo infinitas vías de solución, y que está en dependencia del nivel de desarrollo de la personalidad alcanzado por los estudiantes con un contenido de enseñanza determinado." En ello, como afirman estos autores, la realidad y la solución de problemas vinculadas con esta constituyen una fuente de contradicciones que logre el desarrollo del estudiante. En este contexto se dan varias condiciones que son importantes destacar:

- Los estudiantes tendrán las posibilidades de explicar sus ideas unos a otros, en pequeños grupos o a la totalidad del aula, o utilizar los errores de los estudiantes como vía para indagar sobre sus causas. Esto es esencial en la estimación de proyectos porque de fallar en su futuro profesional los proyectos se atrasan y se afecta la credibilidad de la organización en la cual están insertados. No se debe desestimar lo que dicen y se debe estimular la evaluación individual y colectiva.

- Se integran las más variadas tipologías de evaluaciones como la coevaluación, heteroevaluación y metaevaluación, sobre todo en las decisiones acerca de los modelos a utilizar porque sus cálculos son para tomar decisiones. Un modelo utilizado en una situación inadecuada generalmente se traduce en una decisión equivocada que a su vez repercute en la organización productora de software. Es por ello que la evaluación continua integrando estos tipos de evaluación les permite adquirir experiencia en el rol de gestor de proyectos.

- Se deberá guiar a los estudiantes para que elaboren resúmenes y cálculos determinan esquemas conceptuales de la interrelación entre los modelos de estimación de proyectos y sus pasos.

- Se deberá planificar, orientar y controlar el estudio independiente de forma sistémica, variada; siempre en estrecha relación con la clase que le corresponde a cada estudiante enfatizando en la profundización de los contenidos relacionados con la estimación.

- Los contenidos informáticos serán tratados con un enfoque integrador y generalizador con las asignaturas del perfil y con otros contenidos de la disciplina científica, de modo que facilite su consolidación y otras enfatizando la relación multidisciplinaria. 
Otros autores (Hong, Hwang, \& Tai, 2013; Molnár, Greiff, \& Csapó, 2013) el error debe considerarse como nueva información para elaborar nuevos problemas en el desarrollo del aprendizaje matemático. Este autor afirma que el error señala una evidencia, entre muchos otros, el proceso de construcción del conocimiento. El error apunta a aspectos importantes para el proceso de investigación. No quiere decir que deben errar para aprender, pero que los errores no son necesariamente un problema $y$, si los hubiere, necesitan ser tratados según corresponda.

\section{Diseño de la implementación práctica}

La docencia de Estimación de Proyectos forma parte del llamado currículo propio de la Carrera de Ingeniería Informática que transcurre en el quinto año de la carrera. En Cuba las carreras universitarias tienen un currículo base determinado por el Ministerio de Educación Superior y a este se les añade el currículo propio que responde a las necesidades del territorio donde los futuros profesionales serán insertados. Este currículo propio se divide en dos tipos de asignaturas llamadas optativas y electivas. Las asignaturas optativas el estudiante puede elegir dentro de un grupo de asignaturas propuestas por el departamento docente. Las electivas es un espacio docente dedicado a una asignatura que ellos pueden elegir dentro de cualquier plan de estudios de cualquier carrera de una universidad del país. Los contenidos de estimación de proyectos son parte de las asignaturas optativas por lo cual el nivel de ingreso de los estudiantes es también un indicador interesante de la motivación por aprender este proceso.

La experiencia fue aplicada durante 3 años. Durante el primer año se inscribieron en la asignatura un total de 10 estudiantes de 30 , en el segundo año se inscribieron 24 de 32 y en el último año de la experiencia se inscribieron 28 estudiantes de 28, lo cual demuestra el nivel de aceptación que tuvieron los estudiantes por esta asignatura.

Se impartió docencia durante todo el semestre a un grupo utilizando la integración de los enfoques propuesta. Se asume que el grupo del primer año como G1 así como G2 y G3 a los grupos del segundo y tercer año respectivamente. Es necesario aclarar que cada turno de clases está compuesto por un tiempo de 45 minutos, 5 de receso y 45 minutos siguientes.

En cada grupo se estructuraron tres primeras conferencias orientadoras, la primera para comenzar el tratamiento del proyecto así como las formas en que se procedería durante todo el curso y precisar la evaluación del proyecto a entregar. Se aclaran con los estudiantes la necesidad de poseer un proyecto y se explicitan las formas en que ellos han levantado los requisitos de cada proyecto como punto de partida para la selección del proyecto de cada uno de ellos. Este levantamiento del tipo de proyecto a realizar y la forma de levantamiento de los requisitos permite al profesor corregir los posibles errores en este proceso así como diagnosticar los proyectos a desarrollar de tal manera que le permite precisar los modelos de estimación correctos para cada uno de ellos. Los estudiantes plantean sus dudas al respecto y son aclaradas como parte inicial de la implementación práctica. Se asumieron como principios rectores del curso la ética necesaria para no nombrar profesores en el tratamiento a los contenidos informáticos que se integran de su formación informática como ejemplos y no copiar ejemplos de proyectos de internet. Esta idea de copiar proyectos se les plantea a partir de la inutilidad de copiar proyectos si ellos deben realizar una estimación de sus propios proyectos. Los estudiantes fueron informados en la primera clase de que serían objeto de modificación de los métodos de enseñanza y consintieron en ello.

\section{Materiales y Métodos empleados}


Esta investigación fue conducida a partir de las observaciones en clases, entrevistas a profesores de otras asignaturas y encuestas realizadas a estudiantes del grupo y de otros grupos. En cada clase se fue observando cómo se van apropiando de los contenidos de estimación y los van trasladando a su clase. Las encuestas a los otros estudiantes de años anteriores para analizar la opinión que estos poseen sobre la importancia de la estimación de proyectos. Se aplican la misma cantidad de entrevistas y encuestas en los estudiantes y profesores en el mismo período para contrastar la información. Posteriormente se realizan exámenes finales en los cuales deben presentar y discutir una clase.

La docencia fue estructurada siguiendo los modelos de estimación y sus acciones reveladas en la literatura consultada al respecto. En este caso se comienza por puntos de función por ser la más simple y terminar con SLIM que es más compleja. De la primera clase se orienta el levantamiento de requisitos del proyecto de cada estudiante. Se comienza la segunda conferencia con la estimación por puntos de función tomando como punto de partida el estudio independiente orientado sobre la base de la pregunta ¿cómo puedo saber cuánto es el costo, el tiempo y el esfuerzo que demora en desarrollar sus sistemas? Los estudiantes plantean sus insatisfacciones sobre este acápite en clases recibidas y sobre estas y la revisión del estudio independiente se estructura la primera estimación que ellos realizan. En la misma medida que se va introduciendo los elementos necesarios de esta estimación los estudiantes van corrigiendo los errores cometidos con ayuda de los otros y del profesor. Se orienta como estudio independiente la culminación del proceso de estimación del proyecto respondiendo a la pregunta para qué se va a enseñar ese conocimiento y la revisión de las clases que se encuentran en el EVEA instalado en la universidad. En esta primera etapa se permiten las imprecisiones entre contenido y conocimiento por ser desconocido para los estudiantes.

Posteriormente se estructura el segundo modelo de estimación: COCOMO II apuntando a los estudiantes la necesidad de conocer para qué se va a enseñar. Para introducir este modelo se parte de un proceso de estimación en el cual los puntos de función aportan una estimación no funcional para el gestor de proyecto. Es parte de esta clase las acciones de estimación de estos. Este tratamiento se comienza a partir de la revisión del estudio independiente realizado por los estudiantes y los errores que traen al plantear el objetivo. De esta manera se van introduciendo los modelos de estimación como consecuencia de las fallas del modelo estudiado anteriormente

La mayor cantidad de problemas se pueden observar en los estudiantes están relacionados con los ítems de ponderación y el momento para utilizarlos además de los cálculos. La corrección de estos elementos, teniendo en cuenta las acciones de estimación es necesario abordarlo desde una perspectiva problémica que lleve al estudiante a definirlo correctamente teniendo en cuenta el papel de cada modelo de estimación. En las diferentes clases se van corrigiendo los diversos errores sobre la base de la corrección colectiva de los proyectos de cada uno de los estudiantes. De esta manera, construyendo y reconstruyendo la estimación de los proyectos y su estructura interna se va planificando el proceso que le estudiante debe presentar como proyecto final del curso.

\section{Resultados}

Los resultados obtenidos en las entrevistas a los profesores se reflejan en la tabla \#1 que se muestra a continuación: 


\begin{tabular}{|c|c|c|c|c|c|c|c|c|c|}
\hline \multicolumn{1}{|c|}{ Tabla 1: Resultados de las entrevistas a los profesores } \\
\hline & Primera Entrevista & \multicolumn{2}{|c|}{ Segunda Entrevista } & Tercera Entrevista & \multicolumn{2}{c|}{ Cuarta Entrevista } \\
\hline & Positivo & Negativo & Positivo & Negativo & Positivo & Negativo & Positivo & Negativo \\
\hline Profesores & 1 & 10 & 3 & 8 & 6 & 5 & 9 & 2 \\
\hline & 9,09 & 90,91 & 27,27 & 72,73 & 54,55 & 45,45 & 81,82 & 18,18 \\
\hline
\end{tabular}

Se puede apreciar en los resultados ascendentes en cuanto a la estimación de proyecto a de los estudiantes en el G1. Los estudiantes muestran mayor interés por la estimación de proyectos, prestan mayor atención a los aspectos informáticos y se preparan para las estimaciones con mayor calidad en el estudio independiente.

Los resultados obtenidos en las entrevistas a los profesores se reflejan en la tabla \#2 que se muestra a continuación:

\begin{tabular}{|c|c|c|c|c|c|c|c|c|}
\hline \multicolumn{1}{|c|}{ Tabla 2: Resultado de encuestas a los estudiantes } \\
\hline & Primera Encuesta & \multicolumn{2}{|c|}{ Segunda Encuesta } & \multicolumn{2}{c|}{ Tercera Encuesta } & \multicolumn{2}{c|}{ Cuarta Encuesta } \\
\hline & Positivo & Negativo & Positivo & Negativo & Positivo & Negativo & Positivo & Negativo \\
\hline Estudiantes & 1 & 29 & 18 & 12 & 27 & 3 & 29 & 1 \\
\hline & 1,67 & 48,33 & 30,00 & 20,00 & 45,00 & 5,00 & 48,33 & 1,67 \\
\hline
\end{tabular}

Los resultados permiten apreciar la insatisfacción de los estudiantes con la estimación de proyectos desde el inicio. Existían muchos cuestionamientos acerca de su necesidad. A medida que se fueron introduciendo nuevos contenidos, se sistematizaron las clases y los contenidos de la carrera en la clase que ellos debían planificar los resultados fueron cambiando de negativo en su amplia mayoría a positivo en la generalidad de los estudiantes. Ellos fueron apreciando la necesidad de planificar las actividades de clases para la estimación de los colegas en la futura organización. Esto se reflejó en la práctica laboral de los estudiantes insertados en una organización donde estimaron algunos proyectos sencillos.

De estos resultados se puede triangular la información obtenida resaltando la similitud de apreciación de los profesores y de los estudiantes en cuanto al comportamiento positivo de estos últimos relacionados con la enseñanza de la estimación de proyectos.

En la tabla \#3 se reflejan los resultados de los indicadores declarados acerca de la motivación de los estudiantes. ¡Error! Vínculo no válido.En las observaciones a clases que fueron impartidas a los estudiantes se pudo concluir lo siguiente:

1. La cantidad de estudiantes que integraron la estimación de proyectos en su proyección futura como profesionales en las organizaciones fue en aumento en la medida que el curso fue avanzando. Sin embargo, en los grupos anteriores en los cuales continuaron con los métodos tradicionales se aprecia que los estudiantes en su amplia mayoría continúa sin incluir la estimación de proyectos como parte de su proyección futura.

2. La mayor cantidad de estudiantes que identifican la estimación de proyectos como una de las posibles esferas de actuación como ingenieros informáticos fue incrementándose. Ello permite inferir que cada vez hay una cantidad de estudiantes cada vez menor que rechazan la estimación de proyectos como una posible ocupación. Mientras, en los grupos en los cuales no fue aplicada la experiencia, se observa una situación desfavorable relacionada con este indicador. La contrastación entre los grupos permite afirmar que la integración de los enfoques propuesta permite que los estudiantes aprecien la estimación 
de proyectos como una herramienta de trabajo que puede ser incorporada a su perfil ocupacional.

3. El análisis del comportamiento del indicador relacionado con la autonomía e iniciativa se puede apreciar el incremento sostenido de los estudiantes a los cuales se les aplicó la propuesta y un comportamiento similar a los anteriores indicadores en los restantes grupos. Se llega a conclusiones similares de los indicadores anteriores. En este indicador es necesario destacar que el traslado de situaciones nuevas al proyecto que deben presentar y los niveles de independencia con los cuales deben realizarlo ayuda al desarrollo de la iniciativa. Este indicador es de especial relevancia para los ingenieros informáticos pues es la independencia es una de las cualidades que con mayor relevancia se expresa dentro de los objetivos de su formación.

4. Siguiendo el mismo análisis de los indicadores anteriores, los niveles de satisfacción personal de los estudiantes se elevan en el grupo donde se aplicó la propuesta mientras que en los restantes grupos no se logra. Para los estudiantes resultó gratificante la resolución de problemáticas relacionadas con la estimación de proyectos, se manifestaron vivencias relacionadas con sus experiencias docentes y fueron criticados en las formas como se conducian hasta el momento con sus colegas.

5. El traslado de contenidos de una situación alejada del proyecto que se está realizando para aplicarla es un indicador de apropiación activa y consciente de estos contenidos. Otro aspecto también lo constituye la evaluación de los proyectos de otros colegas en el aula. En este orden se constata un aumento significativo de los estudiantes con niveles de desarrollo alto de este indicador mientras que la situación en el resto se mantiene de la misma manera que con otros indicadores.

Otro aspecto necesario de destacar es las evaluaciones docentes realizadas. En todos los grupos se estructuró la evaluación final a partir de la presentación de la estimación del proyecto que les corresponde. Los niveles de satisfacción del tribunal evaluador con los resultados del grupo en el cual se aplicó la propuesta se expresan en los resultados: 20 estudiantes con la máxima calificación, 8 estudiantes con buenas calificaciones y sólo dos estudiantes con calificaciones regulares. Parte de esta evaluación de la propuesta se incluyeron los resultados en el la Jornada Científica Estudiantil de Ciencias Técnicas de la Universidad donde los estudiantes presentan sus estimaciones preparadas. En esta jornada obtuvo el primer lugar un estudiante del G1. Fue además seleccionado para las jornadas municipales y provinciales obteniendo primer lugar en todas.

\section{Conclusiones}

El aprendizaje de la estimación de proyectos es un proceso en el cual se adolecen de serias insuficiencias de preparación que no existen en las carreras de formación pedagógicas. La selección de los contenidos de enseñanza relacionados con la estimación de proyectos en el contexto de estos profesionales debe ser orientado a las necesidades de su profesión de tal manera que se logre una integración multidisciplinar.

Se fundamentó la integración de los enfoques problémico, de sistema y el de proyectos deben asumir la estimación de proyectos como eje conductor e integrador del proyecto que logra la motivación en estos estudiantes. La estructura interna las estimaciones es el conocimiento que logra aglutinar, en un primer momento, el resto del sistema de conocimientos del cual debe apropiarse el estudiante. Conjuntamente 
con ello, las estimaciones de proyectos como proceso formativo también es parte del contenido de enseñanza.

La implementación práctica de la alternativa propuesta arrojó resultados que permiten aseverar la validez de la misma. La cantidad de estudiantes en los cuales se desarrollaron los indicadores que caracterizan la variable motivación fueron incrementándose durante el proceso de aprendizaje de la estimación de proyectos.

\section{Referencias}

Autores, C. (2003). Pensando en la personalidad (L. F. Rius Ed.). Ciudad de la Habana: Editorial Félix Varela.

Biafore, B. (2011). Successful Project Management Applying Best Practices and Real-World Techniques with Microsoft@ Project. USA: O’Reilly Media.

Dawson, C. W. (2009). Projects in computing and information systems : a student's guide (Second Edition ed.): Addison-Wesley.

Expósito Ricardo, C. (2009). Elementos de Matodología de la Enseñanza de la Informática. Ciudad de la Habana: Editorial Pueblo y Educación.

González-Jorge, H., Roca, D., Torres, S., Armesto, J., \& Puente, I. (2014). Una experiencia de Aprendizaje basado en Proyectos en el ámbito tecnológico: Diseño de un sistema de navegación indoor de bajo coste. Revista de Formación e Innovación Educativa Universitaria., 7(1).

González Hernández, W. (2004). Metodología para contribuir al desarrollo de la creatividad en estudiantes de la educación superior a través de la enseñanza de la programación. (Doctor en Ciencias Pedagógicas.), Universidad Pedagógica "Enrique José Varona". , Academia de Ciencias de Cuba.

González Hernández, W. (2013a). Creativity Development in Informatics Teaching Using the Project Focus. iJEP, 3(1), $22-30$.

González Hernández, W. (2013b). Creativity Development in Informatics Teaching Using the Project Focus. International Journal of Engineering Pedagogy (iJEP), 3(1), pp. 63-70.

González Hernández , W. (2013). Intuition as Part of Informatics Creativity. iJEP, 3(3), 7.

González Hernández, W., \& Estrada Sentí, V. (2014). El desarrollo de la creatividad en la enseñanza de la informática a través del enfoque de proyectos. Paper presented at the COMPUMAT, Ciudad de la Habana.

González Hernández, W., Estrada Sentí, V., \& Martínez Llantada, M. (2006). El enfoque de sistema en la enseñanza de la Informática para el desarrollo de la creatividad Revista Enseñanza Universitaria, 32, 45 - 56.

González Rey, F. (2010). Las categorías de sentido, sentido personal y sentido subjetivo en una perspectiva históricocultural: un camino hacia una nueva definición de subjetividad. Universitas Psychologica, 9(1), 241 - 253.

Guitart, M. E. (2011). Del "Aprendizaje Basado En Problemas" (ABP) al "Aprendizaje Basado En La Acción” (ABA). Claves para su complementariedad e implementación. Revista de Docencia Universitaria, 9(1), 91 - 107.

Hanafy Morsy, M., Ali Alsareii, S., Miree Al-Qahtani, J., Hassan Alshiek, M., Saeed AlAyed, M., \& Abdullah, M. (2015). Credit Hours Policy - Is It Working for Hybrid Problem-Based Learning Curriculum: An Experience of Najran School of Medicine KSA. Journal of Research in Medical Education \& Ethics, 5(2), 129-133.

Hong, J.-C., Hwang, M.-Y., \& Tai, K.-H. (2013). Applying the BaGua to revitalize the creative problem solving process during a goal oriented contest. Thinking Skills and Creativity, 9(0), 120-128.

Kanhime Kasavuve, M., \& González Hernández, W. (2014). Evaluación desarrolladora de los conocimientos matemáticos para la formación de profesores de matemática en la provincia de Kuando Kubango, Angola. Revista Didasc@lia: Didáctica y Educación, V(5), 15 - 40.

Lin, C.-J. (2012). An Investigation of Elementary School Students' Positive Thinking, Personality and Creativity. 
López, M. S., \& Vázquez, O. L. V. (2013). Aprendizaje Colaborativo basado en proyectos desarrollados en Ingeniería. Revista Iberoamericana para la Investigación y el Desarrollo Educativo, 10.

McGibbon, C., \& Belle, J.-P. V. (2015). Integrating environmental sustainability issues into the curriculum through problem-based and project-based learning: a case study at the University of Cape Town. Current Opinion in Environmental Sustainability, 16, 81 - 88.

Medina, L. Q. (2012). Elementos del desarrollo de la enseñanza problémica en la enseñanza de matemáticas y materias afines: caso de Cuba, Colombia Y México. Revista Caribeña de Ciencias Sociales, 2(1), 35 - 56

Molnár, G., Greiff, S., \& Csapó, B. (2013). Inductive reasoning, domain specific and complex problem solving: Relations and development. Thinking Skills and Creativity, 9(0), 35-45.

Oman, S. K., Tumer, I. Y., Wood, K., \& Seepersad, C. (2013). A Comparison of Creativity and Innovation Metrics and Sample Validation Through in- class Design Projects.

Parra Castrillón, J. E., Castro Castro, C., \& Amariles Camacho, M. (2014). Casos de éxito de la aplicación de la metodología de aprendizaje basado en problemas abp. Revista de Investigación de la Facltad de Ingeniería, $1,12-23$

Peña, M. L. M., Garrido, E. D., \& López, J. M. S. (2015). Coordinación interdisciplinar mediante aprendizaje basado en problemas. Una aplicación en las asignaturas dirección de producción y estadística empresarial. . Revista de Investigación Educativa, 33(1), 163 - 178.

Pressman, R. S. (2010). Software engineering: A practitioner's approach. (Seventh Edition ed.). New York: McGrawHill.

Rivera Julio, Y., \& Turizo Martínez, L. (2014). Abp (aprendizaje basado en problemas) para la enseñanza y el desarrollo de proyectos tecnológicos interdisciplinares basados en arduino. Paper presented at the Encuentro Internacional de Educación en Ingeniería ACOFI 2014, Cartagena de India, Colombia.

Rodríguez González, C. A., \& Fernández Batanero, J. M. (2015). Aplicación y validación de un Aprendizaje Basado en Problemas en estudiantes universitarios de Ingeniería de la Construcción. Paper presented at the Actas del 23 Congreso Universitario de Innovación Educativa en las Enseñanzas Técnicas, Valencia.

Sabaté, J. G., \& Valero-García, M. (2012). Hablando sobre Aprendizaje Basado en Proyectos con Júlia. Revista de Docencia Universitaria, 10(3).

Segura Montero, J., \& González Hernández, W. (2015). La habilidad modelar multimedia en los procesos formativos de los Joven Club. Didasc@alia: Didactica y Educación, 6(2).

Velázquez, Á. P., Pérez, M. H., \& Rodríguez, Y. A. (2012). Elementos teóricos de la enseñanza problémica. Métodos y Categorías. Gaceta Médica Espirituana, 14, 34 - 50. 